Check for updates

Cite this: Nanoscale Adv., 2019, 1, 1096

\title{
Surfactant-assisted individualization and dispersion of boron nitride nanotubes $\uparrow$
}

\author{
Ashleigh D. Smith McWilliams, (D) a Carlos A. de los Reyes, (D) a Lucy Liberman, (D) ${ }^{\mathrm{b}}$ \\ Selin Ergülen, ${ }^{a}$ Yeshayahu Talmon, $\mathbb{D}^{\mathrm{b}}$ Matteo Pasquali (D) acdf \\ and Angel A. Martí (D) *aef
}

Boron nitride nanotubes (BNNTs) belong to a novel class of material with useful thermal, electronic and optical properties. However, the study and the development of applications of this material requires the formation of stable dispersions of individual BNNTs in water. Here we address the dispersion of BNNT material in water using surfactants with varying properties. The surfactants were compared based on the quantity of BNNTs dispersed and the quality of the dispersions, as visualized by AFM and cryo-TEM. All surfactants produce dispersions of individualized or small bundles of BNNTs. Of the surfactants tested, high molecular weight, nonionic surfactants suspend the most BNNTs, while ionic surfactants remove the most h-BN impurities. The surfactant dispersions were further characterized by ensemble measurements, such as UV absorption and photoluminescence, dynamic light scattering (DLS), and zeta potential to investigate dispersion stability and quality. These techniques provide a facile strategy for testing future BNNT dispersions. The results of this study reveal that BNNT dispersions in aqueous solution can be tuned to fit a specific application through surfactant selection.

Received 29th October 2018 Accepted 5th December 2018

DOI: $10.1039 / \mathrm{c} 8 \mathrm{na00315 \textrm {g }}$

rsc.li/nanoscale-advances including protective shields, ${ }^{5}$ thermal and mechanical reinforcements for composites, ${ }^{6}$ and biomedical applications. ${ }^{7-10}$

Despite these exceptional properties, research into the production of large-scale BNNT materials has been relatively limited, particularly when compared to their carbon counterparts. This is largely due to the inability to produce large amounts of BNNTs with high purity. In bulk BNNT synthesis, the resulting BNNTs are contaminated with elemental boron and boron nitride impurities, such as hexagonal boron nitride (h-BN). While published purification methods $\mathrm{s}^{\mathbf{1 1}, \mathbf{1 2}}$ are effective at removing boron and small boron nitride impurities, h-BN is much more difficult to remove due to the similarity of its properties with those of BNNTs (Fig. S1 $\dagger$ ).

The ability to fabricate macroscopic materials of BNNTs has also been hindered greatly by the poor dispersibility of BNNTs in all solvents. Researchers have attempted to overcome this barrier in a variety of ways, including covalent/non-covalent functionalization, ${ }^{13-18}$ polymer or peptide wrapping, ${ }^{19-24}$ co-solvent systems, ${ }^{25}$ surfactants, ${ }^{26-28}$ strong acids, ${ }^{12,29}$ and other aromatic and biomolecules., ${ }^{\mathbf{9 1 0}, 30-34}$ One promising way to disperse BNNTs into aqueous solution is through the use of surfactants. Surfactants are amphiphilic compounds that, when used at high enough concentrations (i.e. the critical micelle concentration), form micelles in solution. While the hydrophobic portion of the surfactant, typically a long carbon chain, will interact with or wrap around the BNNT, the hydrophilic portion will interact with water molecules. This allows the BNNTs to form a dispersion in water, stabilized by either electrostatic (ionic) or steric (nonionic) 
forces. Compared to other dispersion systems, surfactants have some clear advantages. They are inexpensive, can be easily utilized in a wide range of applications, and do not cause any disruption to the $\mathrm{sp}^{2}$ hybridization of the BNNTs.

Recent studies have shown that surfactants such as ammonium-oleate $^{26}$ and sodium dodecylbenzenesulfonate (SDBS) ${ }^{27}$ can yield dispersions of BNNTs in aqueous media; however, these studies did not report the stability of these dispersions with the application of centrifugation nor quantified the amount of material suspended. Without these properties being addressed, it is impossible to compare one dispersion to another or to determine the best surfactant for a particular application. In order to move forward the purification of BNNTs and the fabrication of macroscopic materials, it is necessary to better understand the surfactant properties that enable BNNT dispersion and produce the best quality dispersions of BNNTs into solution. In 2003, Moore and coworkers performed such a study, comparing the dispersion ability of various surfactants and polymers, with single-walled CNTs (SWCNTs). ${ }^{35}$ They utilized UV-visible-NIR absorbance, photoluminescence, and cryo-TEM to study the SWCNT dispersions. Other groups have further investigated these and similar dispersions utilizing additional techniques, such as AFM, DLS, and zeta potential. ${ }^{36-40}$ As the polarity of interatomic bonding changes significantly with the switch from $\mathrm{C}$ to $\mathrm{BN}$ in the nanotubes, we ask how the interactions between the surfactant molecules and BNNTs compare with those of CNTs.

Here we report a systematic study of BNNT dispersion into aqueous solutions of various surfactants; we define a standard procedure for BNNT dispersion and analysis, and report the stability, quality, and concentration of these dispersions via mass conversion measurements, AFM, cryo-TEM imaging, UVVis absorption, fluorescence spectroscopy, DLS, and zetapotential measurements. The eight surfactants selected are commonly used for the dispersion of nanomaterials $\mathrm{s}^{35,37,39-41}$ and have properties that allow for direct comparison. The techniques utilized here could be applied to further expand the surfactant scope in the future.

\section{Experimental}

\section{Materials}

Boron nitride nanotubes (type P1 $\beta$ ) were purchased from BNNT, LLC and surfactants were purchased from BASF (Pluronic F108, F87, and 17R4), TCI America (DTAB and CTAC), Acros (CTAB), and Sigma Aldrich (SDS, Pluronic L81). BNNTs were purified by a method modified from one previously reported by Chen et al. ${ }^{11}$

\section{Instrumentation}

Thermogravimetric analysis (TGA) was performed with a Q-600 Simultaneous TGA/DSC from TA Instruments. AFM measurements were performed with a Bruker Multimode 8 AFM system in tapping mode using ScanAsyst Air silicon cantilevers. CryoTEM specimens were imaged with a FEI Talos 200C highresolution TEM at an accelerating voltage of $200 \mathrm{kV}$ below $-175{ }^{\circ} \mathrm{C}$, using a Gatan 626 cryo-holder. The specimens were studied in the low-dose imaging mode to reduce electron beam radiation damage. Images were recorded digitally by a FEI Falcon III direct-imaging camera and the TIA software, with the help of the "phase plates" (FEI) to enhance image contrast. ${ }^{\mathbf{4 2 , 4 3}}$ Absorbance measurements were acquired using a Shimadzu 2450 UV-Visible spectrophotometer. Photoluminescence spectra were measured with a Horiba Nanolog Spectrophotometer. The samples were excited at $250 \mathrm{~nm}$ through a $5 \mathrm{~nm}$ slit and recorded from 290 to $450 \mathrm{~nm}$ with a slit width of $5 \mathrm{~nm}$. DLS and zeta potential measurements were obtained using a Malvern Zen 3600 Zetasizer with the dispersions injected into disposable polystyrene cuvettes and folded capillary cells respectively. All measurements were conducted at $25{ }^{\circ} \mathrm{C}$ and at the natural $\mathrm{pH}$ of the surfactant solution. For zeta potential measurements, the sample was dialyzed in Cellu-Sep H1 cellulose tubular membranes (MWCO: 2000) for 6 hours to remove excess surfactant.

\section{Preparation of dispersions}

Approximately $8 \mathrm{mg}$ BNNTs were added to a vial with $8 \mathrm{~mL}$ of $1 \mathrm{wt} \%$ surfactant solution. The solution was bath ultrasonicated for 10 minutes (Cole-Parmer 8891, $42 \mathrm{kHz}$ ) and then centrifuged at $12000 \mathrm{~g}$ for 30 minutes (with glass inserts in the Eppendorf tube). The supernatant was collected in a separate vial, for imaging and spectroscopy, and the pellet was redispersed in water. The redispersed pellet was collected in a preweighed vial, dried under vacuum at $75{ }^{\circ} \mathrm{C}$, and the final mass recorded.

\section{Determination of mass conversion}

The dried pellet consisting of BNNTs and surfactant was analyzed by thermogravimetric analysis (TGA). The samples were heated to $115{ }^{\circ} \mathrm{C}$, kept at that temperature for $20 \mathrm{~min}$ to remove any remaining water, and then heated to $1000{ }^{\circ} \mathrm{C}$ at a rate of $15^{\circ} \mathrm{C} \mathrm{m^{-1 }}$ under $100 \mathrm{ml} \mathrm{min}^{-1}$ air flow. As organic compounds burn around $200-400{ }^{\circ} \mathrm{C}$ and BNNTs are thermally stable until $>900{ }^{\circ} \mathrm{C}$ (Fig. 1), the percentage of the total mass due to BNNTs can be easily determined. By multiplying the minimum mass percentage (measured at $700{ }^{\circ} \mathrm{C}$ ) by the total mass of the pellet, we determined the final mass of BNNTs in the pellet, and, by extension, the amount of BNNTs that were transferred into the supernatant. We then divide the calculated mass of BNNTs dispersed in solution by the initial mass of BNNTs added to the solution to obtain the percent mass conversion.

\section{Preparation of control sample}

Approximately $8 \mathrm{mg}$ BNNTs were added to a preweighed vial with $1 \mathrm{~mL}$ of $1 \mathrm{wt} \%$ surfactant. This solution was briefly sonicated, dried under vacuum at $75^{\circ} \mathrm{C}$, and its final mass recorded. The final solid was analyzed by TGA. As no centrifugation or transfer steps were undertaken, the final mass conversion should be around $0 \%$.

\section{Preparation of AFM and cryo-TEM samples}

The supernatant from the BNNT dispersions were collected for imaging. AFM samples were prepared by depositing the BNNT 


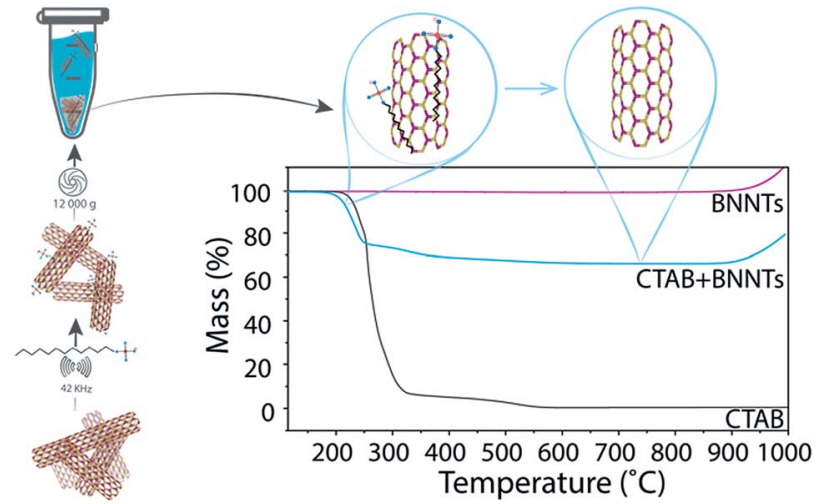

Fig. 1 Schematic demonstrating how the experiment is performed (left) and TGA profiles of BNNTs, CTAB, and a BNNT-CTAB pellet (right). Samples containing $C T A B$ experience a drop in mass from $200-400{ }^{\circ} \mathrm{C}$ due to burning of the organic surfactant. BNNTs are oxidized above $900{ }^{\circ} \mathrm{C}$, resulting in a rapid increase in mass. The minimum mass $\%$ (at $700^{\circ} \mathrm{C}$ ) is used to determine the mass of BNNTs in the sample.

dispersion on a freshly cleaved mica surface (primed with $\mathrm{MgCl}_{2}$ ) using a drop-casting method, washing off excess surfactant with water, and drying in the oven. Cryo-TEM specimens were prepared in a controlled environment vitrification system (CEVS) ${ }^{44}$ at $25{ }^{\circ} \mathrm{C}$ and $100 \%$ relative humidity to prevent water evaporation from the specimen. A drop (ca. $3 \mu \mathrm{L})$ of the dispersion was applied onto a perforated carbon film supported on a copper TEM grid, blotted with a filter paper for thin film $(<300 \mathrm{~nm})$ formation, and plunged into liquid ethane at its freezing point for vitrification..$^{\mathbf{4 4 5}}$

\section{Measurement of BNNT bundle size by AFM}

AFM images were processed in Gwyddion and the height profiles of 100 randomly selected nanotubes and bundles were collected for each sample.

\section{Preparation of dispersions with different concentrations of SDS}

Five SDS solutions were prepared with concentrations of 0.06 , $0.12,0.236,0.5$, and $1.0 \mathrm{wt} \%$ respectively. Approximately $5 \mathrm{mg}$ BNNTs were added to a vial with $5 \mathrm{~mL}$ of each SDS solution. The solution was bath ultrasonicated for 10 minutes and then centrifuged at $12000 \mathrm{~g}$ for 30 minutes. The supernatant was collected, diluted $7 \times$ with water, and the UV-Vis absorbance measured. The absorbance of BNNTs at $204 \mathrm{~nm}$ (after subtracting the absorbance of free SDS solution at the same concentration) and the extinction coefficient were used to calculate the mass conversion for each sample.

\section{Testing dispersion stability}

Dispersions were prepared, as detailed above, in SDS, CTAC, and Pluronic F108. The supernatant was collected and split into two portions. UV-Vis absorbance and DLS measurements were taken on Days $0,1,3,5,7,14,21$, and 28. For each absorbance measurement, $0.5 \mathrm{~mL}$ of the concentrated sample was added to
$3 \mathrm{~mL}$ of DI water. The absorbance was measured from 190 to $400 \mathrm{~nm}$ with $0.5 \mathrm{~nm}$ resolution. The absorbance of free surfactant solution diluted by the same amount was subtracted, and the resulting absorbance at $204 \mathrm{~nm}$ was recorded and tracked over time. For DLS measurements, approximately $1 \mathrm{~mL}$ of the supernatant was added to a disposable polystyrene cuvette and the mean hydrodynamic radius was recorded. DLS measurements were also taken of free surfactant solution, but the hydrodynamic radius was found to be insignificant in all cases.

\section{Testing different centrifugation rates}

Approximately $8 \mathrm{mg}$ of BNNTs were added to $8 \mathrm{~mL}$ of $1 \mathrm{wt} \%$ $\mathrm{CTAB}$ and sonicated for 10 minutes. The solution was then centrifuged for 30 minutes at $1000 \mathrm{~g}, 3000 \mathrm{~g}, 6000 \mathrm{~g}$, or $12000 \mathrm{~g}$ or allowed to settle overnight $(1 g)$. The supernatant of each sample was imaged by AFM and analyzed by DLS and zeta potential measurements, as described above.

\section{Results and discussion}

Eight surfactants with varying properties, such as charge and molecular weight, were tested for their ability to disperse BNNTs. In each test, BNNTs were added to a $1 \mathrm{wt} \%$ solution of surfactant in a $1 \mathrm{mg} \mathrm{mL} \mathrm{mL}^{-1}$ concentration. After sonication (10 $\mathrm{min}, 42 \mathrm{kHz}$ ) and centrifugation (30 min at $12000 \mathrm{~g}$ ), the mass conversion of BNNTs into solution (percent of BNNTs in solution from the original amount added to the mixture) was calculated. Because absorbance of many surfactants overlaps considerably with that of BNNTs, absorbance measurements could not be utilized to determine BNNT concentration in solution. A new method was developed using thermogravimetric analysis (TGA), which takes advantage of the thermal stability of BNNTs when compared to organic surfactants. The precipitate after centrifugation was collected, dried and analyzed by TGA to determine the weight corresponding to surfactant. Then, since the initial amount of BNNTs and the amount left in the precipitate were known, the amount of BNNTs dispersed in solution could be easily determined. The mass of BNNTs in the supernatant was not measured directly, as the very small mass of BNNTs remaining in solution $(\leq 0.8 \mathrm{mg})$ would have produced a large relative error. A schematic portraying the experimental details and TGA results for BNNTs with cetyltrimethylammonium bromide (CTAB) is presented in Fig. 1.

Control TGA experiments are performed on samples that do not undergo a centrifugation step and the water is just evaporated. Because all the material is still in the sample, the mass conversion of control samples is expected to be around $0 \%$. The mass conversion measured for each surfactant is displayed in Fig. 2 and compared to that reported for SWCNTs ${ }^{35}$ and the control (for values see Table S1 $\dagger$ ).

The mass conversions for BNNT material ranged from 0$10 \%$. Of the surfactants tested, it appears that, as was seen with SWCNTs, the highest molecular weight (MW), nonionic surfactant (Pluronic F108) was the most effective for BNNT dispersion. Pluronic F108 (MW $=14600 \mathrm{~g} \mathrm{~mol}^{-1}$ ) showed a similar mass conversion for BNNTs (10\%) and SWCNTs 


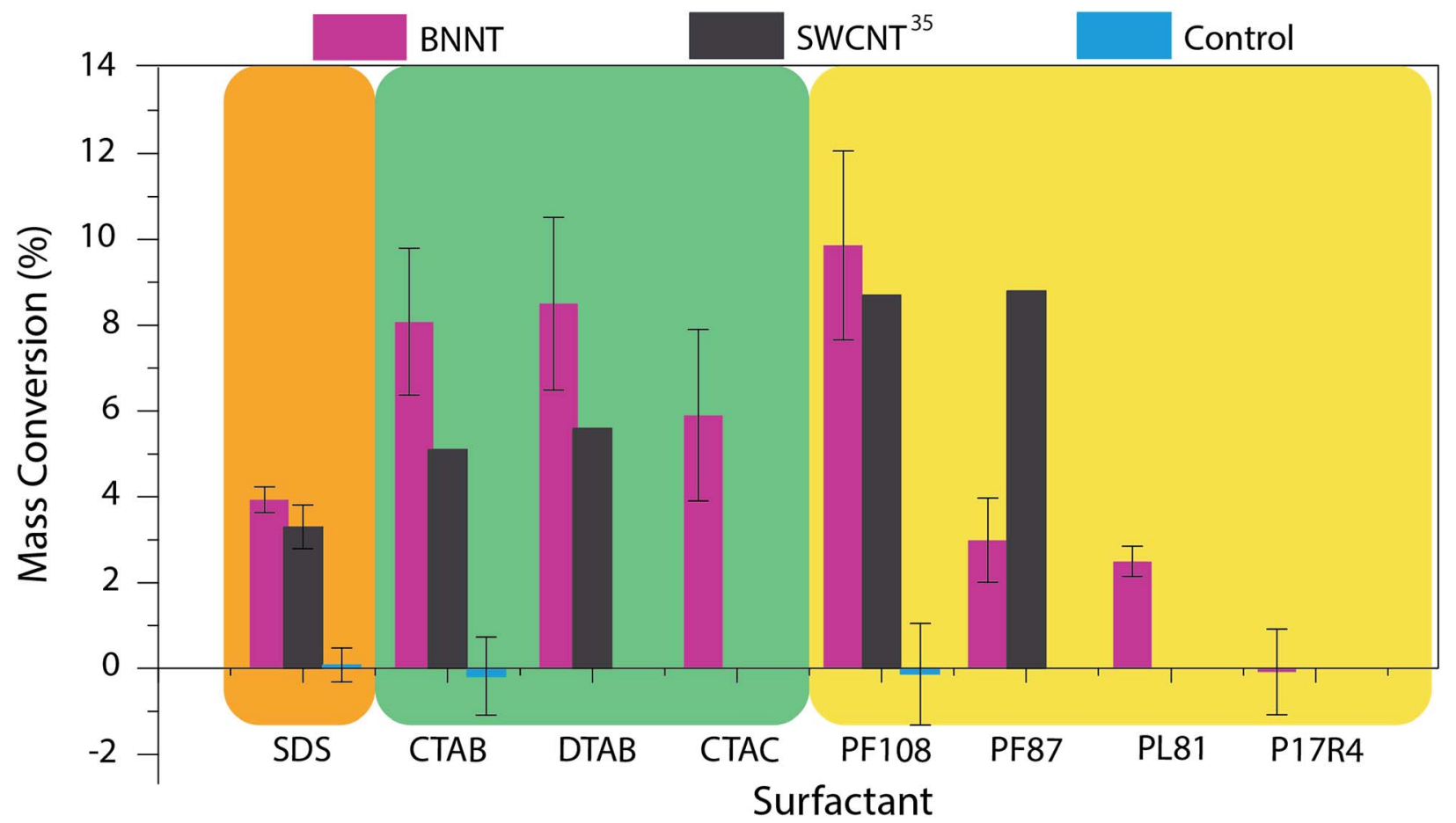

Fig. 2 Mass conversion of BNNTs dispersed in eight sample surfactants. The results were compared to those obtained by Moore and coworkers for SWCNTs ${ }^{35}$ and control experiments. Surfactant structures and images of the produced dispersions can be found in Fig. S2 and S3 respectively. (SDS = sodium dodecyl sulfate; CTAB = cetyltrimethylammonium bromide; DTAB = dodecyltrimethylammonium bromide; CTAC $=$ cetyltrimethylammonium chloride; PF108 = Pluronic F108; PF87 = Pluronic F87; PL81 = Pluronic L81; P17R4 = Pluronic 17R4; Pluronic ${ }^{\circledR}=$ poly(ethylene oxide)-poly(propylene oxide)-poly(ethylene oxide) (PEO-PPO-PEO) triblock copolymer (F108, F87, L81) 46,47 $^{\circ}$ or PPO-PEO-PPO triblock copolymer (17R4) $\left.{ }^{48}\right)$.

(8.7\%). However, this mass conversion dropped from $10 \%$ to $3 \%$ with the switch to Pluronic F87 (MW $\left.=7700 \mathrm{~g} \mathrm{~mol}^{-1}\right)$. This occurred at a higher MW for BNNTs than was witnessed for SWCNTs; a similar drop was seen for SWCNTs (from $8.7 \%$ to $2.5 \%)$ in Pluronic F77 $\left(\mathrm{MW}=6600 \mathrm{~g} \mathrm{~mol}^{-1}\right) .{ }^{35}$ This decline in mass conversion could also be attributed to the decrease in polyethylene oxide (PEO) composition from $80 \%$ to $70 \%$ (ref. 46) as this reduction increases the hydrophobicity of the polymer. Further reduction in the polymer's molecular weight and PEO composition, however, showed very little change in mass conversion: in Pluronic L81 (MW $\left.=2800 \mathrm{~g} \mathrm{~mol}^{-1}, 10 \% \mathrm{PEO}\right)^{47}$ BNNTs had a mass conversion of $2.5 \%$. Moore and coworkers report no conversion for SWCNTs in Pluronic polymers with molecular weights less than $4620 \mathrm{~g} \mathrm{~mol}^{-1}$, however the exact species tested were not reported. We further tested the importance of the order of the triblock copolymer. While Pluronic F108, F87, and L81 are composed of a PEO-PPO-PEO structure, Pluronic 17R4 ( $\mathrm{MW}=2700 \mathrm{~g} \mathrm{~mol}^{-1}$ ) has the hydrophobic component on the exterior (PPO-PEO-PPO). ${ }^{\mathbf{4 8 4 9}}$ Unsurprisingly, BNNTs could not be dispersed using Pluronic 17R4.

Focusing on the ionic surfactants; anionic surfactant, SDS, has a slightly, though insignificantly, higher mass conversion for BNNTs $(3.9 \%)$ than for SWCNTs (3.3\%). However, cationic surfactants, CTAB and DTAB, are much more effective at dispersing BNNTs. Mass conversion for both CTAB and DTAB was around $8 \%$ for BNNTs as compared to $c a$. 5\% for SWCNTs. We believe that this increase in dispersion ability could be due to the partial negative charge on the nitrogen atoms of the BNNTs, leading to an increased interaction between the surfactant and the BNNTs. Unlike the trend seen with nonionic surfactants, however, there is not a significant change in mass conversion with the decrease in aliphatic chain length from 16 to 12 carbons. In addition to testing chain length for cationic surfactants, the impact of the surfactant's counter ion was also studied. The shift

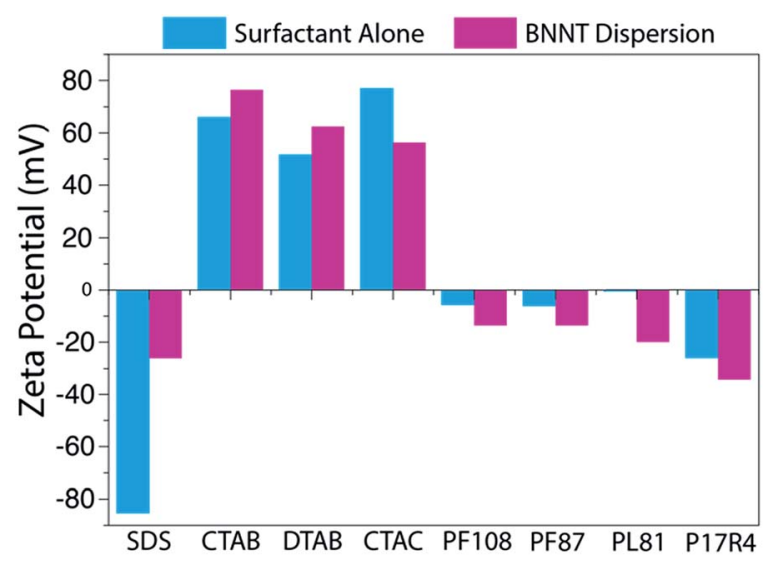

Fig. 3 Zeta potentials measured for each surfactant and surfactantBNNT dispersion. The zeta potential reveals the degree of electrostatic repulsion between nearby particles and is an indicator of dispersion stability. The chart reveals a similar trend, among ionic surfactants, to that seen for their mass conversion. 
from a bromide (CTAB) to chloride (CTAC) counter ion showed a slight, though not significant, decrease in dispersion ability.

Zeta potential measurements were obtained for each sample to help assess the quality of the dispersions (Fig. 3). Each sample was dialyzed prior to measurement in order to remove excess surfactant and decrease the contribution of free surfactant to the zeta potential, which could shadow the values of the dispersed BNNTs. As was expected from previous work with SWCNT-surfactant dispersions, ${ }^{39,40}$ the zeta potential of dispersions in ionic surfactants trends well with the mass conversion results observed, while the nonionic surfactants show no trend (Fig. 3). In the case of dispersions that rely on electrostatic interactions, more stable dispersions should produce a larger magnitude zeta potential. We observed that our CTAB-BNNT dispersion, which had one of the highest mass conversions of the ionic surfactants, produced the largest magnitude zeta potential $(77 \mathrm{mV})$, while SDS, which showed the lowest mass conversion, produced the smallest $(26 \mathrm{mV})$.

In addition to comparing the quantity of BNNTs in the dispersions, we studied the individualization of BNNTs and whether h-BN impurities (which are present in the starting material - Fig. S1 $\dagger$ ) were effectively removed from the dispersion. We used AFM to obtain good height resolution and a large overview of the sample, and cryo-TEM for high-spatial resolution examination of the solutions in their native state. Images were collected for BNNTs dispersed in SDS, CTAB, and Pluronic F108, as these showed the highest mass conversion for their respective category (Fig. 4, S4 $\dagger$ ). All three surfactants yielded individualized or small bundles of BNNTs, with primarily individualized tubes in CTAB and Pluronic F108. Additionally, in the case of SDS and CTAB, the images appear clean and without significant h-BN or other impurities. On the contrary, while Pluronic F108 shows a higher density of BNNTs, the sample appears to contain a small fraction of h-BN sheets alongside the nanotubes.

AFM images were additionally used to obtain detailed bundle size information. 100 nanotubes or bundles were randomly selected and their heights measured for each surfactant dispersion. For all surfactants tested, the average bundle size was below $10 \mathrm{~nm}$ and at least 95\% of those measured had a height less than $20 \mathrm{~nm}$, further confirming that a large majority of the BNNTs were individualized or in fewnanotube aggregates (Fig. S5 $\dagger$ ).

The BNNT-surfactant dispersions were also characterized spectroscopically. Unfortunately, the ultraviolet absorption and emission of BNNTs (Fig. 5) overlaps considerably with many surfactants, making their study cumbersome. Additionally, we found that most common laboratory plasticware (such as microcentrifuge tubes and pipette tips) contributes to fluorescence impurities in this region. We solved these problems by studying BNNTs in SDS and using only glassware for BNNT processing. As SDS contributes minimally to absorbance and emission in the UV region, it can be easily subtracted as background. Also, glass does not contribute to fluorescence
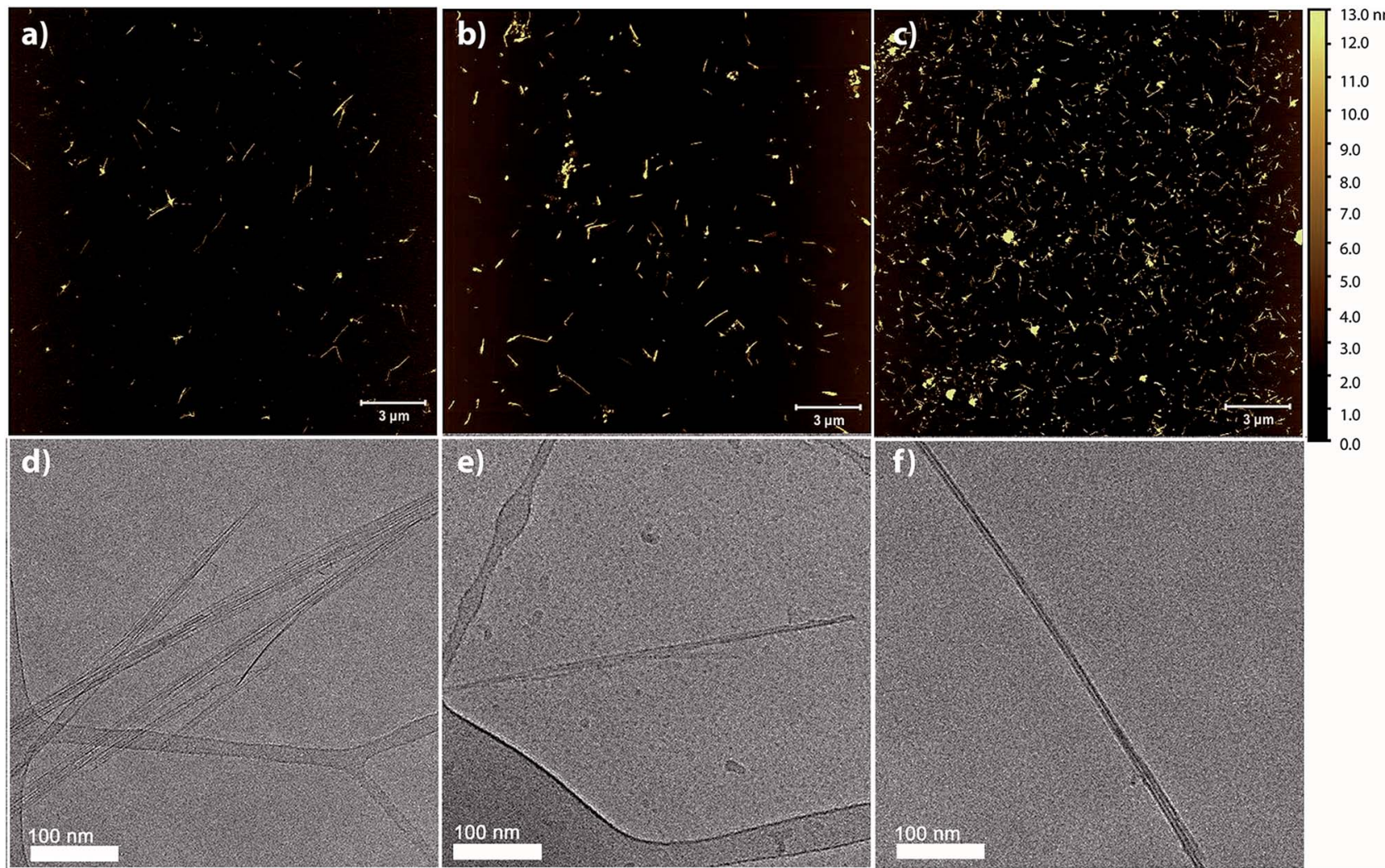

Fig. 4 AFM and cryo-TEM images of BNNT dispersions in SDS ( $a, d)$, CTAB (b, e), and Pluronic F108 (c, f). The images reveal that the BNNTs in our dispersions are primarily individualized or in small bundles. 


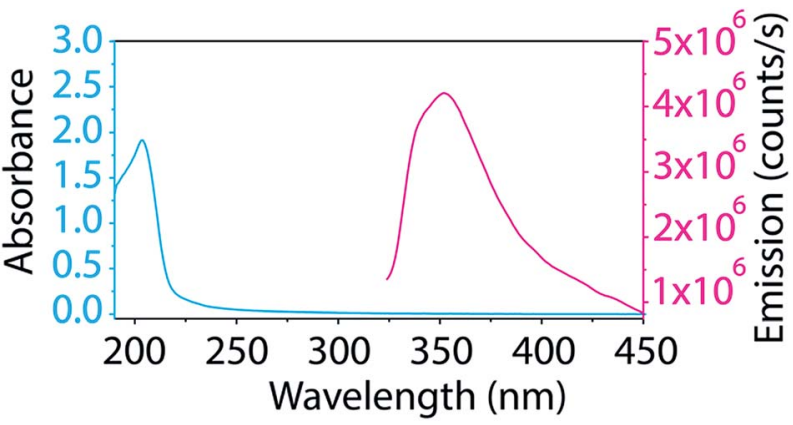

Fig. 5 Absorption and emission spectra of BNNTs dispersed in SDS show a sharp absorption peak at $204 \mathrm{~nm}$ and a broad fluorescence emission around $352 \mathrm{~nm}$.

impurities, so glass inserts and pipettes prevent contamination. The supernatant from BNNT dispersions in SDS was utilized for absorption and photoluminescence measurements. The BNNTs dispersed in SDS produce a sharp absorption peak at $204 \mathrm{~nm}$ and a broad fluorescence emission with a maximum at $352 \mathrm{~nm}$ (Fig. 5). This emission has been previously attributed to bandto-band optical transitions across the direct band gap. ${ }^{26,50}$ Taking advantage of the minimal absorption at $204 \mathrm{~nm}$ of SDS, we determined the extinction coefficient of BNNTs in SDS to be

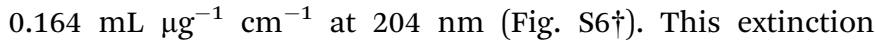
coefficient can be conveniently used as a tool for determining the concentration of BNNTs in SDS.

Using this newly determined extinction coefficient for BNNTs in SDS, we studied how the change in surfactant concentration affects the dispersion. The surfactant concentration ( $1 \mathrm{wt} \%$ ) used for this study was chosen originally due to its observed effectiveness at dispersing CNTs. ${ }^{36,40,51,52}$ In fact, Sun et al. found this concentration of surfactant to be optimum for dispersing CNTs in an array of surfactants. ${ }^{40}$ Additionally, this concentration falls well above the critical micelle concentration (CMC) for all surfactants tested. However, to ensure that the results observed for CNTs would hold true for BNNTs, we studied the mass conversion of BNNTs with different

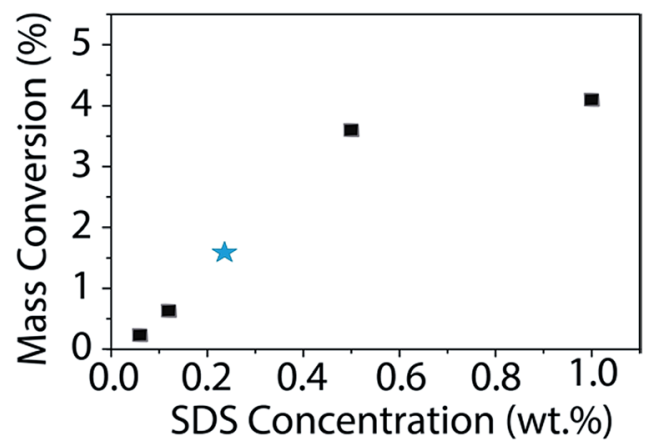

Fig. 6 Mass conversion (\%) of BNNTs as a function of SDS concentration. The initial nanotube concentration is $1 \mathrm{mg} \mathrm{mL}^{-1}$. The published CMC for SDS is $0.236 \mathrm{wt} \%$ (ref. 53) and is denoted with a blue star. BNNT mass conversion quickly increases once the concentration of SDS is above the CMC with a maximum of approximately $4 \%$ conversion in $1 \mathrm{wt} \%$ SDS. concentrations of SDS (Fig. 6). We utilized the calculated extinction coefficient of BNNTs in SDS to quickly determine the mass conversion in each SDS concentration. As was observed by Sun et al. for CNTs, BNNT mass conversion is low at concentrations below the CMC, but quickly increases and reaches a plateau as surfactant concentration increases, with little difference going from 0.5 to $1 \mathrm{wt} \%$ SDS.

Beyond characterizing the BNNT-surfactant dispersions just after being prepared, we wanted to ensure that they remained stable over time. We utilized absorbance and DLS measurements to track the state of the dispersions over one month. UV absorbance will provide information about the relative concentration of nanotubes in solution, and therefore allow us to determine if material is crashing from solution overtime (Fig. 7a). On the other hand, DLS measures the hydrodynamic radius of the particles, and would inform us if the material is aggregating in solution (Fig. 7b). We investigated BNNT dispersions in SDS, CTAC, and Pluronic F108, as these surfactants had the least overlap in absorbance with that of BNNTs. Over the month-long timeframe we saw no significant changes in the UV absorbance or hydrodynamic radius, leading us to conclude that the dispersions are stable over time.

Finally, we investigated how centrifugation rate affected BNNT dispersion quality. CTAB-BNNT dispersions prepared with five different centrifugation rates were studied by AFM, DLS, and zeta potential measurements. As expected, the average
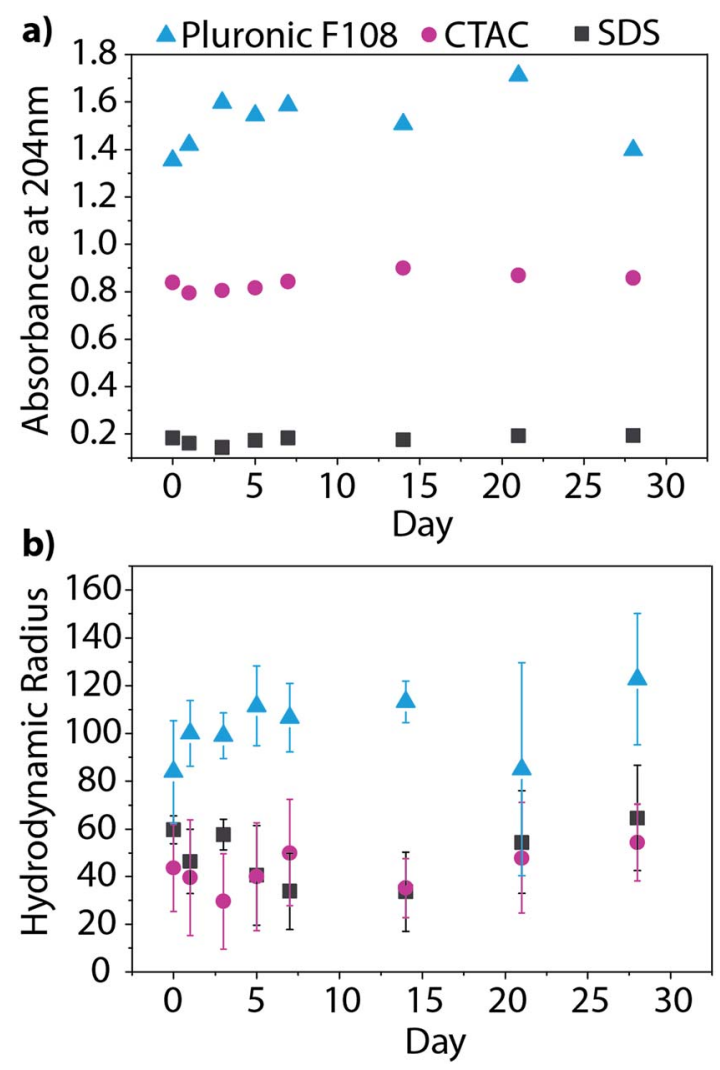

Fig. 7 BNNT dispersions in SDS, CTAC, and Pluronic F108 tracked over one month by (a) UV absorbance and (b) DLS. Both measurements show no significant changes over time confirming that the dispersions are stable. 

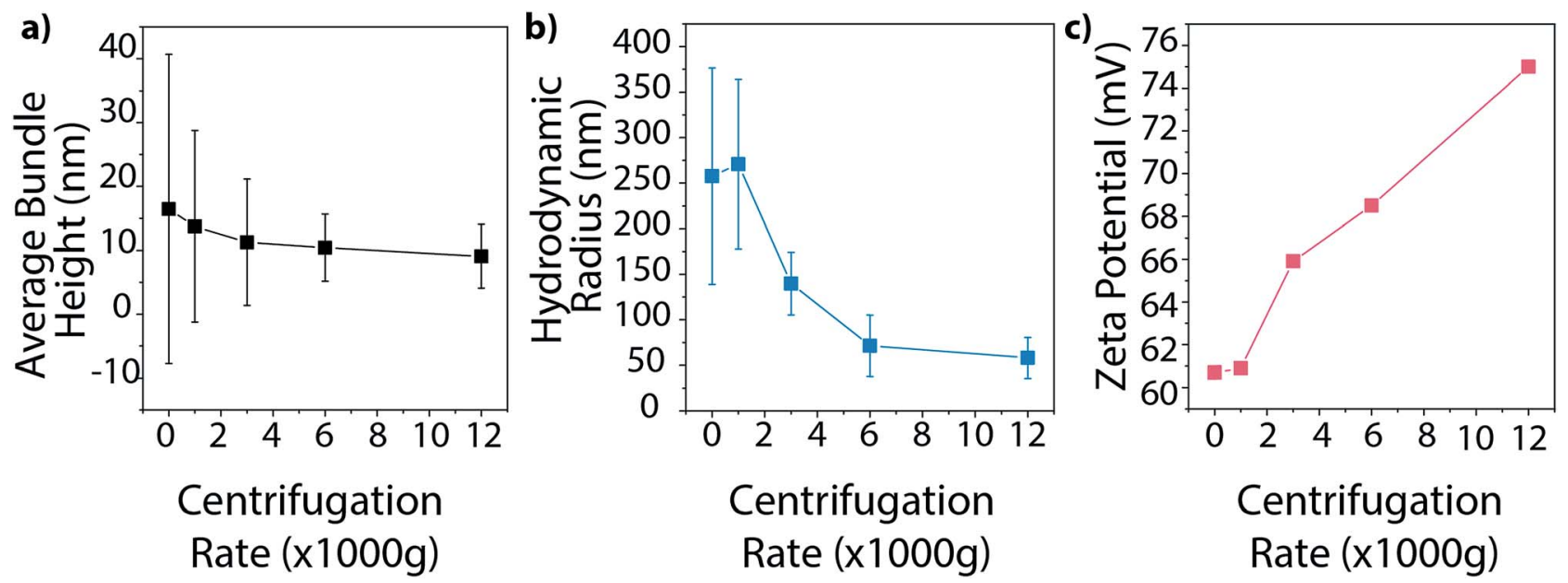

Fig. 8 Tracking the impact of centrifugation rate on the (a) average BNNT bundle height measured using AFM, (b) hydrodynamic radius, and (c) zeta potential of BNNTs dispersed in CTAB. Increasing centrifugation rate produces smaller and more uniform dispersions with increased stability.

bundle size, as measured by AFM, decreased with increasing centrifugation rates as larger aggregates were forced out of solution (Fig. 8a, histograms in Fig. $55 \dagger$ ). The average hydrodynamic radius, as measured by DLS, followed the same trend, revealing a large drop in aggregate size with the increase in centrifugation force from 1000 to $6000 \mathrm{~g}$, followed by a minor decrease at $12000 \mathrm{~g}$ (Fig. 8b). Both methods further demonstrate a decrease in size distribution with increasing centrifugation rate. Finally, zeta potential measurements increase with increasing centrifugation rate (Fig. 8c). Since zeta potential is linked with electrostatic dispersion stability, we can conclude that increasing the centrifugation rate, and removing larger aggregates of BNNTs, produces more stable dispersions.

\section{Conclusions}

BNNTs possess many exceptional properties that make them ideal for a wide range of applications. However, difficulties in purification and low dispersibility in all solvents makes them challenging to work with, and has hindered the production of BNNT-based macro-materials. By performing a systematic study of BNNT dispersion in surfactants, we have gained insight into what properties make their dispersion optimal. Particularly, we have found that high molecular weight, nonionic surfactants are the most effective at dispersing BNNTs, producing a high concentration of mostly individualized tubes. However, they are not as specific in their dispersion when compared to ionic surfactants, so more h-BN impurities are also found remaining in solution. Conversely, while ionic surfactants produced a smaller overall mass conversion, imaging revealed that they dispersed nanotubes much more specifically, producing dispersions with fewer impurities. Finally, though SDS showed the smallest mass conversion, SDS is basically transparent in the UV, with little absorption or fluorescence, which makes it ideal for spectroscopic studies. The BNNT concentration in SDS can also be readily determined using a calculated extinction coefficient. BNNT dispersions were found to be stable over one month by UV and DLS tracking and various techniques were used to confirm the optimum surfactant concentration and centrifugation rate for the dispersion of BNNTs. These results demonstrate that BNNT dispersions in aqueous solution can be tuned to fit a specific application through adjustments in surfactant selection. Additionally, the use of ensemble techniques, such as UV absorbance and zeta potential measurements, can provide simple and efficient ways to compare dispersion quality. From the production of composites to biomedical applications, the ability to suspend individual nanotubes and easily modify their dispersion properties will unlock many potential applications for BNNTs.

\section{Conflicts of interest}

There are no conflicts to declare.

\section{Acknowledgements}

We acknowledge the NSF (CHE 1610175 and 1807737), AFOSR (FA9550-18-1-0014), US-Israel Binational Science Foundation (2012223, 2016161), and the Welch Foundation (C-1668) for their financial support. Cryo-TEM was performed at the Technion Laboratory for Electron Microscopy of Soft Materials, supported by Technion Russell Berrie Nanotechnology Institute (RBNI). We thank Thomas Dushatinski (BNNT, LLC) for helpful discussions and assistance procuring material for study.

\section{Notes and references}

1 N. G. Chopra and A. Zettl, Solid State Commun., 1998, 105, 297-300.

2 C. H. Lee, S. Bhandari, B. Tiwari, N. Yapici, D. Zhang and Y. K. Yap, Molecules, 2016, 21, 922.

3 D. Golberg, Y. Bando, Y. Huang, T. Terao, M. Mitome, C. Tang and C. Zhi, ACS Nano, 2010, 4, 2979-2993. 
4 X. Blase, A. Rubio, S. G. Louie and M. L. Cohen, Europhys. Lett., 1994, 28, 335-340.

5 Y. Li, P. S. Dorozhkin, Y. Bando and D. Golberg, Adv. Mater., 2005, 17, 545-549.

6 C. Zhi, Y. Bando, C. Tang, H. Kuwahara and D. Golberg, Adv. Mater., 2009, 21, 2889-2893.

7 J. Niskanen, I. Zhang, Y. Xue, D. Golberg, D. Maysinger and F. M. Winnik, Nanomedicine, 2016, 11, 447-463.

8 X. Chen, P. Wu, M. Rousseas, D. Okawa, Z. Gartner, A. Zettl and C. R. Bertozzi, J. Am. Chem. Soc., 2009, 131, 890-891.

9 A. Rocca, A. Marino, S. Del Turco, V. Cappello, P. Parlanti, M. Pellegrino, D. Golberg, V. Mattoli and G. Ciofani, Biochim. Biophys. Acta, Gen. Subj., 2016, 1860, 775-784.

10 S. Del Turco, G. Ciofani, V. Cappello, M. Gemmi, T. Cervelli, C. Saponaro, S. Nitti, B. Mazzolai, G. Basta and V. Mattoli, Colloids Surf., B, 2013, 111, 142-149.

11 H. Chen, Y. Chen, J. Yu and J. S. Williams, Chem. Phys. Lett., 2006, 425, 315-319.

12 M. Adnan, D. M. Marincel, O. Kleinerman, S.-H. Chu, C. Park, S. J. A. Hocker, C. Fay, S. Arepalli, Y. Talmon and M. Pasquali, Nano Lett., 2018, 18, 1615-1619.

13 C. A. de los Reyes, K. L. Walz Mitra, A. D. Smith, S. Yazdi, A. Loredo, F. J. Frankovsky, E. Ringe, M. Pasquali and A. A. Martí, ACS Appl. Nano Mater., 2018, 1, 2421-2429.

14 C. Zhi, Y. Bando, C. Tang, S. Honda, K. Sato, H. Kuwahara and D. Golberg, Angew. Chem., Int. Ed., 2005, 44, 7932-7935.

15 C. Y. Zhi, Y. Bando, T. Terao, C. C. Tang, H. Kuwahara and D. Golberg, Chem.-Asian J., 2009, 4, 1536-1540.

16 S.-Y. Xie, W. Wang, K. A. S. Fernando, X. Wang, Y. Lin and Y.-P. Sun, Chem. Commun., 2005, 3670-3672.

17 W. Wang, Y. Bando, C. Zhi, W. Fu, E. Wang and D. Golberg, J. Am. Chem. Soc., 2008, 130, 8144-8145.

18 W.-Q. Han and A. Zettl, J. Am. Chem. Soc., 2003, 125, 20622063.

19 C. Zhi, Y. Bando, C. Tang, R. Xie, T. Sekiguchi and D. Golberg, J. Am. Chem. Soc., 2005, 127, 15996-15997.

20 Z. Gao, C. Zhi, Y. Bando, D. Golberg and T. Serizawa, J. Am. Chem. Soc., 2010, 132, 4976-4977.

21 Y.-T. R. Lau, M. Yamaguchi, X. Li, Y. Bando, D. Golberg and F. M. Winnik, J. Phys. Chem. C, 2013, 117, 19568-19576.

22 C. H. Lee, D. Zhang and Y. K. Yap, J. Phys. Chem. C, 2012, 116, 1798-1804.

23 S. Velayudham, C. H. Lee, M. Xie, D. Blair, N. Bauman, Y. K. Yap, S. A. Green and H. Liu, ACS Appl. Mater. Interfaces, 2010, 2, 104-110.

24 J.-H. Choi, J. Kim, D. Seo and Y.-S. Seo, Mater. Res. Bull., 2013, 48, 1197-1203.

25 A. L. Tiano, L. Gibbons, M. Tsui, S. I. Applin, R. Silva, C. Park and C. C. Fay, Nanoscale, 2016, 8, 4348-4359.

26 J. Yu, Y. Chen and B.-M. Cheng, Solid State Commun., 2009, 149, 763-766.

27 M. Zheng, C. Ke, I.-T. Bae, C. Park, M. W. Smith and K. Jordan, Nanotechnology, 2012, 23, 095703.

28 X. J. Zeng and W. L. Liu, Micro Nano Lett., 2014, 9, 569-571.
29 O. Kleinerman, M. Adnan, D. M. Marincel, A. W. K. Ma, E. A. Bengio, C. Park, S.-H. Chu, M. Pasquali and Y. Talmon, Langmuir, 2017, 33, 14340-14346.

30 Z. Gao, C. Zhi, Y. Bando, D. Golberg, M. Komiyama and T. Serizawa, RSC Adv., 2012, 2, 6200-6208.

31 C. Zhi, Y. Bando, W. Wang, C. Tang, H. Kuwahara and

D. Golberg, Chem.-Asian J., 2007, 2, 1581-1585.

32 D. Kim, T. Sawada, C. Zhi, Y. Bando, D. Golberg and T. Serizawa, J. Nanosci. Nanotechnol., 2014, 14, 3028-3033.

33 M. Emanet, Ö. Şen and M. Çulha, Nanomedicine, 2017, 12, 797-810.

34 A. Salvetti, L. Rossi, P. Iacopetti, X. Li, S. Nitti, T. Pellegrino, V. Mattoli, D. Golberg and G. Ciofani, Nanomedicine, 2015, 10, 1911-1922.

35 V. C. Moore, M. S. Strano, E. H. Haroz, R. H. Hauge, R. E. Smalley, J. Schmidt and Y. Talmon, Nano Lett., 2003, 3, 1379-1382.

36 M. J. O'Connell, S. M. Bachilo, C. B. Huffman, V. C. Moore, M. S. Strano, E. H. Haroz, K. L. Rialon, P. J. Boul, W. H. Noon, C. Kittrell, J. Ma, R. H. Hauge, R. B. Weisman and R. E. Smalley, Science, 2002, 297, 593-596.

37 J. Y. Lee, J. S. Kim, K. H. An, K. Lee, D. Y. Kim, D. J. Bae and Y. H. Lee, J. Nanosci. Nanotechnol., 2005, 5, 1045-1049.

38 M. Yang, M. Li, S. Luo and R. Liang, Int. J. Adv. Des. Manuf. Technol., 2016, 82, 361-367.

39 B. White, S. Banerjee, S. O'Brien, N. J. Turro and I. P. Herman, J. Phys. Chem. C, 2007, 111, 13684-13690.

40 Z. Sun, V. Nicolosi, D. Rickard, S. D. Bergin, D. Aherne and

J. N. Coleman, J. Phys. Chem. C, 2008, 112, 10692-10699.

41 R. J. Smith, M. Lotya and J. N. Coleman, New J. Phys., 2010, 12, 125008.

42 K. Nagayama, Eur. Biophys. J., 2008, 37, 345-358.

43 R. Danev and K. Nagayama, Methods Enzymol., 2010, 481, 343-369.

44 J. R. Bellare, H. T. Davis, L. E. Scriven and Y. Talmon, J. Electron Microsc. Tech., 1988, 10, 87-111.

45 Y. Talmon, J. Mol. Liq., 2015, 210, 2-8.

46 P. Alexandridis and T. Alan Hatton, Colloids Surf., A, 1995, 96, 1-46.

47 T. M. Krupka and A. A. Exner, Int. J. Hyperthermia, 2011, 27, 663-671.

48 B. C. Kumi, B. Hammouda and S. C. Greer, J. Colloid Interface Sci., 2014, 434, 201-207.

49 A. Bodratti and P. Alexandridis, J. Funct. Biomater., 2018, 9, 11.

50 J. Wu, W.-Q. Han, W. Walukiewicz, J. W. Ager, W. Shan, E. E. Haller and A. Zettl, Nano Lett., 2004, 4, 647-650.

51 T. Hertel, A. Hagen, V. Talalaev, K. Arnold, F. Hennrich, M. Kappes, S. Rosenthal, J. McBride, H. Ulbricht and E. Flahaut, Nano Lett., 2005, 5, 511-514.

52 C. Richard, F. Balavoine, P. Schultz, T. W. Ebbesen and C. Mioskowski, Science, 2003, 300, 775-778.

53 P. Mukerjee and K. J. Mysels, Critical micelle concentrations of aqueous surfactant systems, 1971, vol. 36. 